RESEARCH NOTE

\section{Populational Heterogeneity of Brazilian Trypanosoma cruzi Isolates Revealed by the Mini-exon and Ribosomal Spacers}

\author{
O Fernandes/*/+, SS Santos, \\ ACV Junqueira, AM Jansen**, \\ E Cupolillo***, DA Campbell $* * * *$, \\ B Zingales*****, JR Coura
}

Departamento de Medicina Tropical **Departamento de Protozoologia ***Departamento de Imunologia, Instituto Oswaldo Cruz, Av. Brasil 4365, 21045-900

Rio de Janeiro, RJ, Brasil *Departamento de

Patologia, Uerj, Rio de Janeiro, RJ, Brasil ****Department of Microbiology \& Immunology, School of Medicine, University of California, Los Angeles, USA *****Departamento de Bioquímica, Instituto de Química, Universidade de São Paulo, São Paulo, SP, Brasil

Key words: Trypanosoma cruzi - mini-exon ribosomal DNA - major phylogenetic lineages

Chagas disease in humans, a result of infection by the protozoan Trypanosoma cruzi, shows considerable diversity in clinical manifestations and chronic pathology of cardiac or digestive alterations. This variability has been attributed to both variation in the host immune response and to genomic heterogeneity of the parasite. Although described as one taxon, $T$. cruzi shows substantial heterogeneity in genotype and phenotype perhaps as a result of its clonal method of propagation, in which it is proposed that mutations accumulate in different sub-populations of the parasite (M Tibayrenc et al. 1990 Proc Natl Acad Sci USA 87: 2414-2418).

\footnotetext{
${ }^{+}$Corresponding author. Fax: +55-21-280.3740. E-mail: octaviof@gene.dbbm.fiocruz.br

Received 9 June 1999

Accepted 9 August 1999
}

Initial studies using isoenzyme electrophoresis analysis (MA Miles et al. 1977 Trans $R$ Soc Trop Med Hyg 71: 217-225, 1978 Nature 272: 819821, 1980 Trans $R$ Soc Trop Med Hyg 74: 221237, MA Miles \& RE Cibulski 1986 Parasitol Today 4: 94-97) indicated that $T$. cruzi could be classified into three groups (termed zymodemes) based on six enzyme electrophoretic profiles. Zymodeme I and III were associated with forest-dwelling (sylvatic) mammals, such as opossums; zymodeme II was associated with human cases of Chagas disease, domiciliated mammals and domestic triatomines.

Further analysis using additional enzyme markers and larger number of isolates indicated greater diversity within the taxon and 43 zymodemes were defined (M Tibayrenc et al. 1986 Proc Natl Acad Sci USA 83: 115-119, 1993 Proc Natl Acad Sci USA 90: 1335-1339, M Tibayrenc \& FJ Ayala 1988 Evolution 42: 277-292). This increased level of discrimination between $T$. cruzi isolates did not reveal obvious linkages between the zymodemes and aspects of pathology, transmission, epidemiology, or geographic distribution beyond the correlation of zymodemes I, II and III and the epidemiological cycles. The complex structure of $T$. cruzi population, inspired a major effort to determine molecular markers that correlate with specific features of the human-parasite relationship. Similar variability among parasite populations was also observed in restriction-fragment-length polymorphism in the mitochondrial DNA (C Morel \& L Simpson 1980 Am J Trop Med Hyg 29 Suppl: 10701074), nuclear DNA fingerprinting (AM Macedo et al. 1992 Mol Biochem Parasitol 55: 147-154) and karyotyping studies (J Henriksson et al. 1993 Exp Parasitol 77: 334-348, J Henriksson 1996 Parasitol Today 12: 108-114). Once again, no correlation with biological features was observed due to the extreme heterogeneity of the isolates.

In contrast to the diversity suggested by the above techniques, PCR amplification of sequences from the $24 \mathrm{~S} \alpha$ ribosomal RNA (rRNA) gene and from the non-transcribed spacer of the mini-exon gene indicated a clear dimorphism among $T$. cruzi isolates. This dimorphism allowed the definition of two lineages that correlated broadly with zymodemes I and II (O Fernandes et al. $1998 \mathrm{Am} \mathrm{J}$ Trop Med Hyg 58: 807-811, 1999 Parasitology 118: 161-166, RP Souto \& B Zingales $1993 \mathrm{Mol}$ Biochem Parasitol 62: 45-52, RP Souto et al. 1996 Mol Biochem Parasitol 83: 141-152). An examination of 88 T. cruzi stocks collected from humans, wild mammals and triatomines and originating from different Countries of South America (Brazil, Argentina, Chile, Bolivia and Venezuela) by 
mini-exon gene and 24S $\alpha$ rRNA typing approach, and randomly amplified polymorphic DNA (RAPD) analysis further defined two major parasite lineages that represent substantial phylogenetic divergence (RP Souto et al. $1996 \mathrm{Mol}$ Biochem Parasitol 83: 141-152).

To verify a possible association of epidemiological parameters or disease potential of the isolates with the two $T$. cruzi lineages that nowadays are named $T$. cruzi I and T. cruzi II, we also used the mini-exon and/or 24S $\alpha$ rRNA typing method to analyze 86 T. cruzi field stocks (68 isolated from humans and 18 from triatomines) derived from four Brazilian geographic areas. These parasite samples were also clustered into the aforementioned two lineages. The data are suggestive of a preferential association of to human isolates. T. cruzi I to the sylvatic cycle of the parasite and T. cruzi II to human isolates. Furthermore a clear correlation could be made with the morbidity of the disease: areas with high morbidity present the circulation of $T$. cruzi II; T. cruzi $\mathrm{I}$ is evidenced in areas where Chagas disease is infrequent and the morbidity, as evaluated by the level of abnormal electrocardiograms, is low (Fernandes et al. 1998 loc. cit.).

In the wake of these molecular epidemiological studies, an intriguing question remains. How could a parasite change its genome during the transition from the sylvatic (T. cruzi I) to the domestic transmission cycle (T. cruzi II)? To clarify this specific point, we must re-evaluate the sylvatic cycle of T. cruzi in the light of the recent division of this protozoan into the two major lineages. Sixty-eight T. cruzi isolates collected recently from sylvatic mammals and wild bugs from different geographical areas in the State of Rio de Janeiro, a Brazilian region with no cases of autochthonous Chagas disease, were typed. This study revealed that the sylvatic cycle is more complex than previously assumed since both $T$. cruzi lineages were encountered in similar ecotopes (Fernandes et al. 1999 loc. cit.). Therefore, a new proposal for the transmission cycles of $T$. cruzi was elaborated (B Zingales et al. 1998 Inter J Parasitol 28: 105-112).

Our laboratory has also adapted a typing approach (E Cupolilo et al. 1995 Mol Biochem Parasitol 73: 145-155) to be used for T. cruzi strains using trascribed spacers of the ribosomal gene (Fig. 1). Ribosomal RNA genes (rDNA) are highly conserved and have proven to be useful in phylogenetic analysis among trypanosomatids (Cupolilo et al. 1995 loc. cit., O Fernandes et al. $1994 \mathrm{Mol}$ Biochem Parasitol 66: 26221-26271). Typically, in trypanosomatids rDNA is found as tandemlyrepeated units separated by non-transcribed spacers (JL Ramirez \& P Guevara 1987 Mol Biochem Parasitol 66: 261-277, P Guevara et al. $1992 \mathrm{Mol}$ Biochem Parasitol 56: 15-26). Trypanosomatid

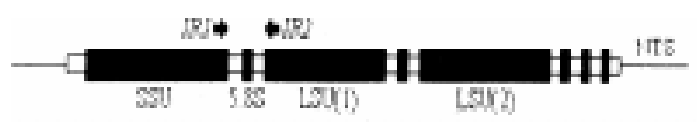

Fig. 1: organization of the trypanosomatid rRNA locus. Arrows indicate the oligonucleotide primers (IR1 and IR2) that anneal to the small subunit (SSU) and large subunit (LSU) respectively. The coding regions, internal transcribed spacer (ITS) and nontranscribed-spacer (NTS) are indicated by filled boxes, empty boxes, or a line, respectively.

rDNA exhibits an unsual organization where the coding regions for the three large and five small ribosomal RNA molecules are separated by internal transcribed spacers (ITS) that show extensive variability. The ITS are relatively small and flanked by highly conserved segments to which PCR primers can be designed. In order to produce an amplification product corresponding to the 5.8S rDNA plus the two flanking ITS, conserved oligonucleotides were used (5'-GCTGTAGGTGAACCTGC AGCAGCTGGATCATT-3' and 5'-GCGGGTAG TCCTGCCAAACACTCAGGTCTG-3'). Amplification reactions were performed as previously described (Cupolilo et al. 1995 loc. cit.) using genomic DNA of 10 T. cruzi strains as templates. Five of them were from Piauí, where T. cruzi II is the most frequent and five from Amazonas, where $T$. cruzi I predominates. The PCR products were further digested by six restrictions enzymes (BstUI, EcoRI, HaeIII, RsaI, Sau3AI and TaqI) and submitted to acrylamide gel electrophoresis (Fig. 2). The PCR products corresponding to the ITS of the T. cruzi isolates from Piauí and Amazonas were distinct in size and the resulting restriction fragment profiles, analyzed by a numerical methodology, generated a phenetic dendrogram that clusters the isolates into the two aforementioned lineages with low level of similarity (Fig. 3). The results show that this approach is also consistent with the sub-division of the taxon T. cruzi into two phylogenetic lineages. Considering the ten isolates that were analyzed, T. cruzi II (Piauí strains - domestic transmission cycle) is less polymorphic than $T$. cruzi I (Amazonas strains- sylvatic transmission cycle). This finding suggests a possible correlation between the complexity of the sylvatic transmission cycle and the diversity of the sylvan parasites (Fernandes et al. 1999 loc. cit.).

Our approaches to the understanding of genetic dimorphism in T. cruzi have focused on two genes, the ribosomal RNA and mini-exon loci. It is clear from karyotype analysis that the same dimorphic nature of the major lineages is evident in other chromosomes (Henriksson 1996 loc. cit.). Furthermore, there is evidence for four or five natural clusterings within $T$. cruzi II (RP Souto et al. Mol 


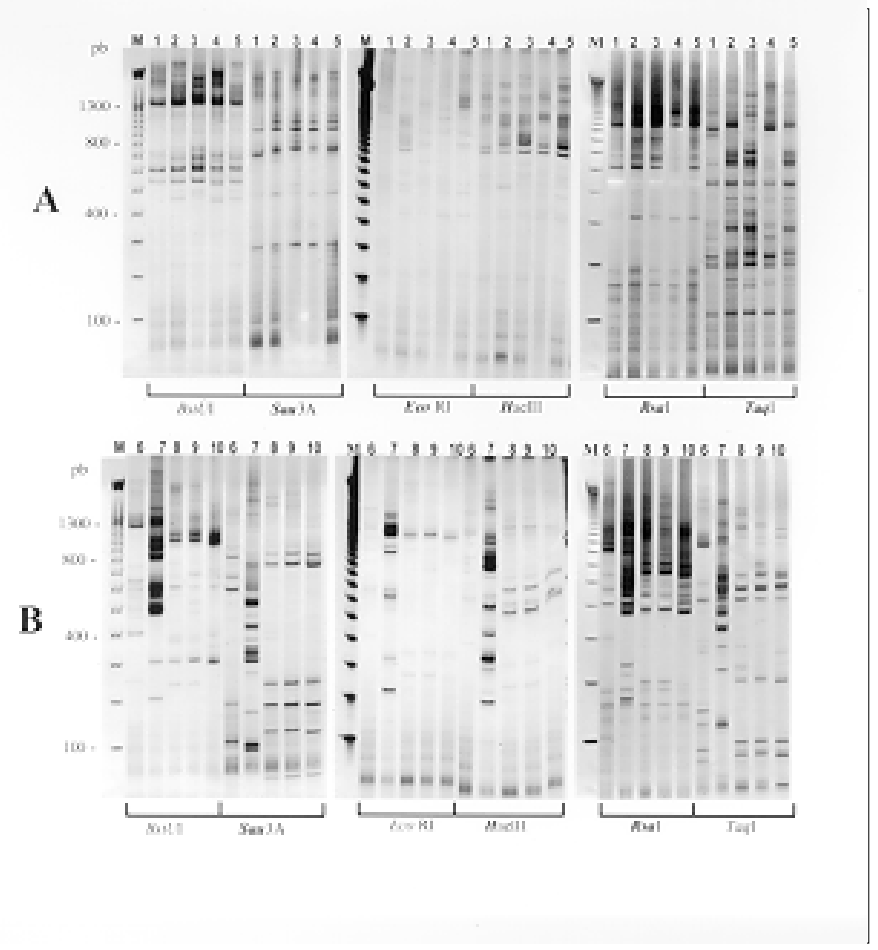

Fig. 2: five percent acrylamide gel electrophoresis showing the restriction enzymes profiles of the internal transcribed spacers (ITS) of the rRNA genes for Trypanosoma cruzi isolates from human patients from (A) Piauí and (B) Amazonas. Molecular marker, 100 base-pair ladder.

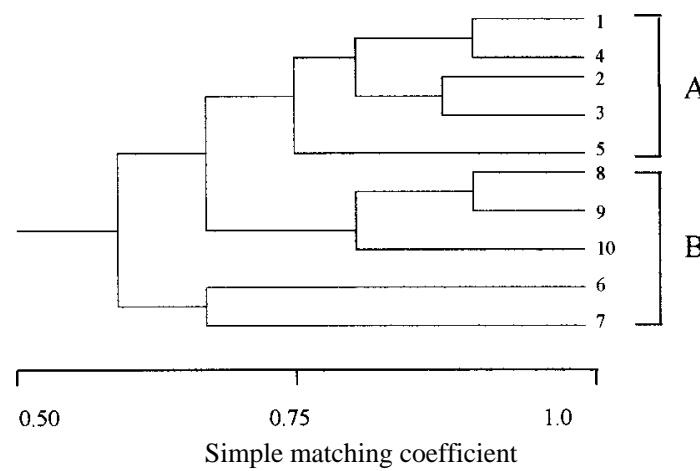

Fig. 3: phenogram showing the similarity among Trypanosoma cruzi isolates. The group A represents the samples from Piauí and the group B the samples from Amazonas.
Biochem Parasitol 83: 141-152, S Brisse et al. 1998 Parasitol Today 14: 178-179). The apparent hetA erozygosity of the rRNA genes within some of these subdivisions (Souto et al. loc. cit.) underscores the need for a more detailed analysis of the genetic constitution of parasites within the taxon T. cruzi. In parallel with the T. cruzi genome project, our future experiments will be directed towards defining other dimorphic markers for additional loci in the T. cruzi genome. Our goal of is to generate markers from all the chromosomes to measure genetic variability between the two lineages. A theoretical "variability" map can be applied to any isolate with known biological/medical history and may eventually yield a correlation to the outcome of infection by $T$. cruzi. 